\title{
Female genital tuberculosis - still a common cause of primary amenorrhea in developing countries
}

\author{
Ramesh Bettaiah, Shraddha Daksha*, Rachana Ghanti, Dhivya Balakrishnan
}

Department of Gynec Laparoscopy, Altius Hospital, Bangalore, India

Received: 26 June 2016

Accepted: 12 July 2016

*Correspondence:

Dr. Shraddha Daksha,

E-mail: drpiddi@gmail.com

Copyright: (c) the author(s), publisher and licensee Medip Academy. This is an open-access article distributed under the terms of the Creative Commons Attribution Non-Commercial License, which permits unrestricted non-commercial use, distribution, and reproduction in any medium, provided the original work is properly cited.

\begin{abstract}
Primary amenorrhea is defined as, no menses by age 14yrs in absence of growth or development of secondary sexual characteristics and no menses by age 16yrs regardless of the presence of normal growth or development of secondary sexual characteristics. Although pulmonary tuberculosis remains the commonest and the most infectious type of tuberculosis, extra pulmonary tuberculosis is becoming more prevalent especially in young women throughout the world. We report a case of young woman presenting as primary amenorrhea apparently having no signs and symptoms of tuberculosis. 20 yrs old unmarried girl was referred for primary amenorrhea with normal secondary sexual characters and presence of uterus, tubes and ovaries on abdominal scan. Hormonal assay and karyotyping was normal. She had negative progesterone challenge test and estrogen progesterone challenge test. Diagnosis of genital tuberculosis was confirmed by diagnostic hysterolaparoscopy and positive tubercular polymerase chain reaction and culture. Hysterolaparoscopy is a key tool for confirmation of diagnosis in cases of primary amenorrhea when the dilemma exists.
\end{abstract}

Keywords: Primary amenorrhea, Female genital tuberculosis (FGTB), Tuberculosis

\section{INTRODUCTION}

Primary amenorrhea is defined as, no menses by age 14 yrs in absence of growth or development of secondary sexual characteristics and no menses by age 16 yrs regardless of the presence of normal growth or development of secondary sexual characteristics. ${ }^{1}$

Incidence of primary amenorrhea due to genital tuberculosis is $2.7 \%$. $^{2}$ Female genital tuberculosis is rare in western world but relevant in developing countries like India. It is the most baffling condition, especially because of its varied presentation. Although pulmonary tuberculosis remains the commonest and the most infectious type of tuberculosis, extra pulmonary tuberculosis is becoming more prevalent especially in young women throughout the world. ${ }^{4}$
Female genital tuberculosis (FGTB) is common in developing countries and associated with significant morbidity in the form of menstrual dysfunction, infertility, tubal block, peritubal adhesions, intrauterine adhesions, and perihepatic adhesions. ${ }^{3,4}$ It is mostly acquired by haematogenous route and always occurs secondary to pulmonary (commonest) or extra pulmonary tuberculosis such as gastrointestinal tract, kidneys, skeletal system, meninges and miliary TB. ${ }^{4}$

Genital tuberculosis frequently presents without symptoms as seen in our case and diagnosis requires high index of suspicion. Fallopian tubes are the first and most commonly affected organ followed by endometrium, ovary and cervix. Adhesions between tubes, ovaries, omentum, intestines, liver and diaphragm are common findings (Fitz Hugh Curtis syndrome). 


\section{CASE REPORT}

20 yrs old unmarried girl presented with isolated primary amenorrhea, without cyclical pain with positive family history of tuberculosis. She was of average build with height $1.55 \mathrm{~m}$ and weight $58 \mathrm{Kg}$. On examination, secondary sexual characters were well developed. Abdomen was soft with no palpable mass. Local examination revealed normal external genitalia. Rectal examination revealed the presence of uterus through rectovaginal septum. Chest x-ray and tuberculin test were negative.

Abdominal and pelvic scan showed normal size uterus with polycystic pattern of the ovaries with no renal anomaly. Hormonal profile including FSH, LH, and TSH, prolactin, testosterone and estradiol were normal. Karyotype was 46XX. Progesterone challenge test and estrogen progesterone challenge test were negative. In view of suspecting end organ problem, diagnostic hysterolaparoscopy was planned.

On hysteroscopy - endometrial cavity was tubular with pale endometrium and uterine synechiae. Both ostia were not visualized. Endometrial sample was taken for histopathology, tubercular polymerase chain reaction and culture.

On laparoscopy - uterus and both ovaries were normal in size. Lead pipe appearance of both the tubes was seen. Left sided periovarian adhesions involving left ovarian fossa and pouch of Douglas were seen. Per hepatic adhesions were seen, suggestive of Fitz Hugh Curtis syndrome. Although omentum appeared to be normal, biopsy was taken. Positive polymerase chain reaction assay and culture reports confirmed the diagnosis of genital tuberculosis. Endometrial biopsy showed chronic non-specific inflammation. Omental biopsy was found to be negative for tuberculosis. Antitubercular treatment was prescribed for six months (2 months of rifampicin, isoniazid, pyrazinamide and ethambutol +4 months of rifampicin and isoniazid).

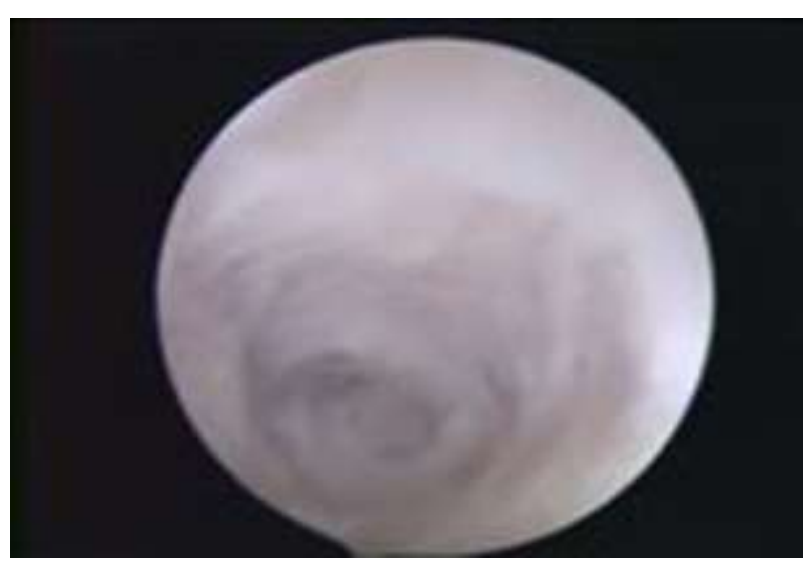

Figure 1: Hysteroscopy showing pale endometrium with tubular cavity and adhesions.

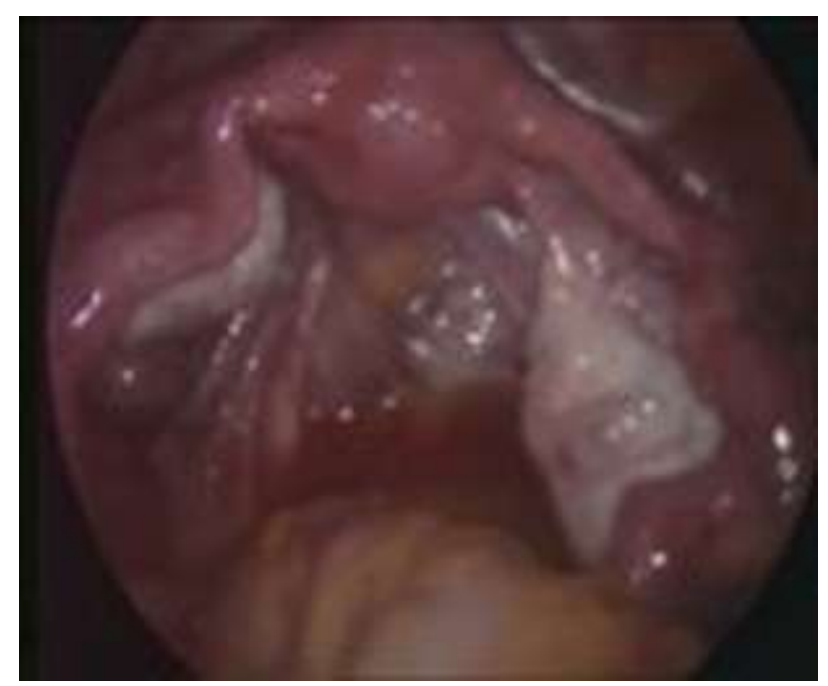

Figure 2: Laparoscopic view of lead pipe appearance of tubes with normal uterus and ovaries.

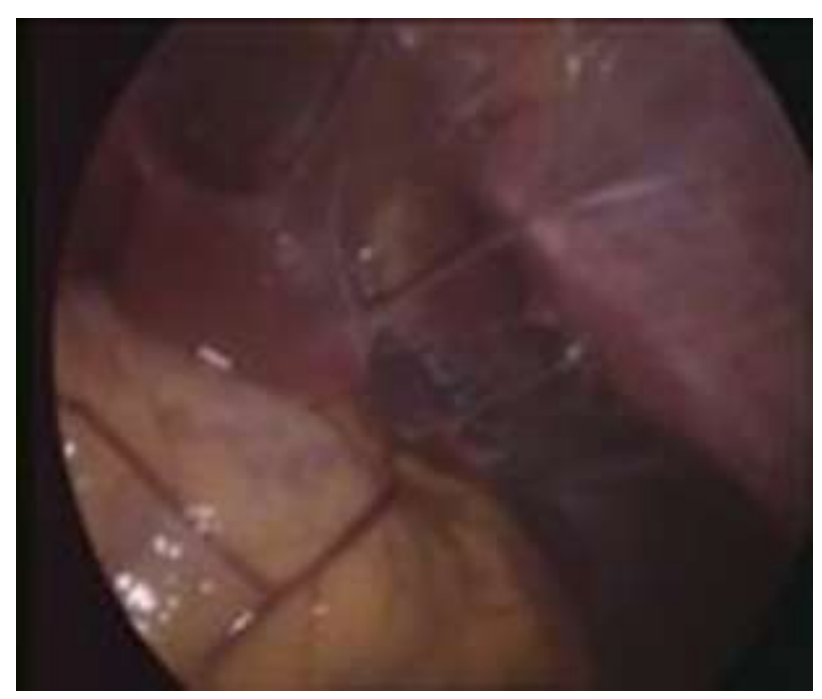

Figure 3: Laparoscopy showing perihepatic adhesions (Fitz-Hugh Curtis syndrome).

\section{DISCUSSION}

Primary amenorrhea due to genital tuberculosis, though very rare, is treatable. Female genital tuberculosis is rare in western world but relevant in developing countries like India. Being a paucibacillary disease, diagnosis of female genital tuberculosis may be difficult., ${ }^{4,5}$ Although gold standard in diagnosis of female genital tuberculosis is demonstration of mycobacterium tuberculosis either on microscopy or culture of endometrial biopsy or presence of epitheloid granuloma on histopathology, they are positive in only few cases leading on to missing of diagnosis in many cases. ${ }^{4,5}$

In the absence of typical epitheloid granuloma and caseation of TB, other features like dilatation of glands, destruction of epithelium and presence of inflammatory exudates in the lumen suggests tuberculous pathology. ${ }^{6}$ 
Past history of TB or family history of TB with chronic inflammatory cells with proliferative endometrium also favours the diagnosis of genital TB. A negative biopsy does not rule out genital TB as TB endometritis is seen in only $50-60 \%$ of cases and there may be sampling error. Polymerase chain reaction (PCR) on endometrial biopsy is a sensitive and rapid method for detecting mycobacterium DNA (MPT 64 gene) but can be false positive and may not be able to differentiate between infection and disease.

Overall, PCR demonstrated M. tuberculosis DNA in 56\% of cases compared to $1.6 \%$ smear positive and $3.2 \%$ culture positive cases. PCR was positive in all women with laparoscopic findings suggestive of $\mathrm{TB}$, in $60 \%$ of those with probable diagnosis, in $33 \%$ of those with incidental findings and even in one case with normal laparoscopic findings. ${ }^{8}$

Hysteroscopy is useful in endometrial disease and may show pale looking endometrium with endometrial cavity being partially or completely obliterated by adhesions of varying grade which may involve ostia. ${ }^{9}$ Total destruction of endometrium may result in Asherman's syndrome resulting in amenorrhea secondary to end organ failure. However, laparoscopy is the most reliable tool to diagnose FGTB, especially for tubal, ovarian, and peritoneal disease. ${ }^{10}$

There can be tubercles on peritoneum or tubes, tubo-ovarian masses, caseous nodules, encysted ascites, various grades of pelvic adhesions, hydrosalpinx, pyosalpinx, beaded tubes, tobacco pouch appearance, and inability to see tubes due to adhesions. ${ }^{11}$

Varying grades of Fitz Hugh Curtis syndrome (violin string adhesions between liver and diaphragm or anterior abdominal wall) is often seen in up to $48 \%$ cases of female genital TB. ${ }^{13}$ Other authors have also found laparoscopy very useful in diagnosis and management of genital and peritoneal TB with the advantage of avoidance of laparotomy which is more traumatic and hazardous especially in a case of abdominopelvic tuberculosis. ${ }^{11,12}$

Multiple drug therapy in adequate doses and for sufficient duration is the mainstay in the treatment of TB including FGTB. Short-course chemotherapy for 6-9 months has been found to be effective for medical treatment of FGTB. Surgical treatment is rarely required and is limited as drainage of pelvic or tubo-ovarian abscesses, pyosalpinx followed by antitubercular treatment for better results. The prognosis of genital tuberculosis for fertility is poor in cases of endometrial and tubal diseases.

\section{CONCLUSION}

Primary amenorrhea is an important gynaecological endocrinal condition that needs proper methodical approach for confirmation of diagnosis. Genital tuberculosis is common in India, a combination of Hysterolaparoscopy along with endometrial histopathological studies, acid fast bacilli culture and polymerase chain reaction provides the best available methods for the diagnosis of female genital tuberculosis in asymptomatic cases.

Patient consent was obtained prior to the study and patient identity not disclosed.

\section{Funding: No funding sources \\ Conflict of interest: None declared \\ Ethical approval: Not required}

\section{REFERENCES}

1. Speroff SL, Fritz MCA. Clinical Gynaecologic Endocrinology and Infertility. edition. 2010:435-494.

2. Khatoon H, Choudhury TA, Mahmud N. Primary amenorrhea: Analysis of 108 Cases. Journal of South Asian Federation of Obstretics and Gynaecology. 2010;2(2):105.

3. World Health Organization. Global Tuberculosis Control. A Short Update to the 2009 Report. WHO/HTM/TB/2009.426. Geneva: WHO; 2009.

4. Sharma JB. Tuberculosis and obstetrics and gynaecological practice. In: Studd J, Tan SL, Chervenak FA, editors. Progress in Obstetrics and Gynaecology. Vol. 18. Philadelphia: Elsevier; 2008. p. 395-427.

5. Neonakis IK, Spandidos DA, Petinaki E. Female genital tuberculosis: A review. Scand J Infect Dis. 2011;43:564-72.

6. Bazaz-Malik G, Maheshwari B, Lal N. Tuberculous endometritis: a clinicopathological study of 1000 cases. Br J Obstet Gynaecol. 1983;90:84-6.

7. Kumar S. Female genital tuberculosis. In: Sharma SK, Mohan A. (eds) Tuberculosis, 1st edn. Delhi, Jaypee. 2001:311-324.

8. Bhanu NV, Singh UB, Chakraborty M, Suresh N, Arora J, Rana T, et al. Improved diagnostic value of PCR in the diagnosis of female genital tuberculosis leading to infertility. J Med Microbiol. 2005;54(Pt10):927-31.

9. Sharma JB, Roy KK, Pushparaj M, Kumar S. Hysteroscopic findings in women with primary and secondary infertility due to genital tuberculosis. Int $\mathrm{J}$ Gynecol Obstet. 2009;104:49-52.

10. Sharma JB, Roy KK, Pushparaj M, Kumar S, Malhotra N, Mittal S. Laparoscopic findings in female genital tuberculosis. Arch Gynecol Obstet. 2008;278:359-64.

11. Jindal UN, Bala Y, Sodhi S, Verma S, Jindal S. Female genital tuberculosis: Early diagnosis by laparoscopy and endometrial polymerase chain reaction. Int J Tuberc Lung Dis. 2010;14:1629-34.

12. Volpi E, Calgaro M, Ferrero A, Viganò L. Genital and peritoneal tuberculosis: Potential role of laparoscopy in diagnosis and management. J Am Assoc Gynecol Laparosc. 2004;11:269-72. 
13. Sharma JB, Roy KK, Gupta N, Kumar S, Malhotra $\mathrm{N}$, Mittal S. High prevalence of Fitz-Hugh-Curtis syndrome in female genital tuberculosis. Int $\mathrm{J}$ Gynaecol Obstet. 2007;99:62-3.

Cite this article as: Bettaiah R, Daksha S, Ghanti R, Balakrishnan D. Female genital tuberculosis - still a common cause of primary amenorrhea in developing countries. Int J Reprod Contracept Obstet Gynecol 2016;5:2891-4. 